\title{
El campo del periodismo de educación en Chile: visiones y prácticas de periodistas y editores/as
}

\section{The Field of Education Journalism in Chile: Visions and Practices of Journalists and Editors}

O campo do Jornalismo Educacional no Chile: visões e práticas de jornalistas e editores

Sebastián Ansaldo, Universidad de Cambridge, Cambridge, Reino Unido (sa850@cam.ac.uk)

RESUMEN | En los últimos años, el periodismo de educación ha emergido como un nuevo tipo de periodismo especializado en Chile. Utilizando la teoría de campos de Bourdieu, junto con elementos de los estudios de mediatización, el artículo busca caracterizar al Periodismo de Educación en Chile explorando sus particularidades, dinámicas internas y relaciones con campos circundantes. Mediante entrevistas a 24 periodistas y editores que cubren temas educativos, realizadas en 2018, se exploran prácticas y visiones de los periodistas, para describir motivaciones, rutinas y percepciones sobre su trabajo e influencia desde y en otros campos. Se concluye que existen diferencias en el nivel de autonomía del campo en función del tipo de contenido, que los periodistas que cubren temas educativo-pedagógicos tienen niveles más altos de capital simbólico, y que los campos del periodismo y educativo tienen cruces e influencias entre ellos.

PALABRAS CLAVE: periodismo de educación; teoría de campos; mediatización; Bourdieu. 
ABSTRACT|In recent years, education journalism has emerged as a new type of specialized journalism in Chile. Using Bourdieu's fields theory and elements of mediatization studies, the paper aims to characterize education journalism in Chile by exploring its particularities, internal dynamics, and relationships with surrounding fields. Through interviews with 24 journalists and editors who cover educational issues, conducted in 2018, I explore practices and visions of journalists, to describe motivations, routines and perceptions about their own work and influence in other fields. The study concludes that there are differences in levels of autonomy in the field depending on the type of content. In addition, journalists who cover educational-pedagogical topics have higher levels of symbolic capital, and there are influences between the journalistic and educational fields.

KEYWORDS: education journalism; fields theory; mediatization; Bourdieu.

RESUMO|Nos últimos anos o jornalismo educacional surgiu como um novo tipo de jornalismo especializado no Chile. Usando a teoria do campo de Bourdieu, juntamente com elementos dos estudos de midiatização, o estudo busca caracterizar o jornalismo educacional no Chile, explorando suas particularidades, dinâmicas internas e relações com os campos circundantes. Através de entrevistas com 24 jornalistas e editores que tratam temas educacionais, realizadas em 2018, são exploradas práticas e visões de o jornalistas sobre educação para descrever motivações, rotinas e percepções sobre seu próprio trabalho, e influências em outros campos. O estudo conclui que há diferenças no nível de autonomia do campo, dependendo do tipo de conteúdo; que os jornalistas que cobrem temas educacionais-pedagógicos possuem níveis mais elevados de capital simbólico; e que os campos do jornalismo educacional têm cruzamentos e influências entre eles.

PALAVRAS-CHAVE: jornalismo educacional; teoria do campo; midiatização; Bourdieu. 


\section{INTRODUCCIÓN}

Después de la irrupción del movimiento estudiantil chileno expresado en las movilizaciones de 2006 y 2011, el rol de los medios en la discusión pública sobre educación se intensificó y expandió (Bellei et al., 2014; Bellei \& Cabalín, 2013). Las redes sociales y los medios tradicionales aumentaron la cobertura de temas educativos (Cabalín, 2014a), con noticias centradas en el movimiento, sus demandas, la respuesta política y la estructura del sistema educativo chileno. Hubo, asimismo, periódicos que crearon secciones especiales sobre educación (Santa Cruz \& Cabalín, 2018).

Algunos comentaristas, políticos, líderes sociales y académicos presentaron reparos a la cobertura del movimiento estudiantil chileno, por no ser equilibrada, centrarse en la criminalización y encuadrarlo sobre todo desde temas como seguridad y orden público. (Figueroa, 2013; Arredondo, 2016; Cabalín, 2013; Saavedra Utman, 2019). Una crítica similar se dirigió a la cobertura mediática del diseño e implementación de reformas derivadas del movimiento, fundamentada en cómo la lógica mediática afectó la política educativa, mediante discursos que mercantilizan la educación y ponen el énfasis en la competencia (Cabalín, 2015).

Los sesgos en la cobertura periodística en educación se han estudiado utilizando principalmente análisis de discurso, visual y de contenido (Cabalín, 2013; Cabalín, 2015; Cabalín \& Antezana, 2016; Cabalín et al., 2019). Estos autores concluyen que en las noticias, editoriales e imágenes de ciertos medios predomina una visión política-económica neoliberal, con una marcada agenda ideológica y que enmarcan la educación como un problema técnico-coyuntural y no en su dimensión políticoestructural. Sin embargo, salvo el trabajo de Santa Cruz y Cabalín (2018), el punto de vista de los profesionales sobre la producción de noticias educativas en Chile y sus características ha sido poco explorado. Estudios de este tipo pueden ser útiles para entender cómo el campo educativo y el periodístico se influyen mutuamente.

Esta investigación se centra en la dinámica del campo del periodismo de educación en Chile y en cómo periodistas y editores perciben su trabajo de producción de noticias en periódicos chilenos, radios y sitios web de noticias. Explora cómo este proceso comparte algunas de las principales características del periodismo, pero dadas las particularidades de la agenda educativa y la naturaleza del campo, es un tipo de periodismo único y particular. Su naturaleza depende, en gran medida, del tipo de medio y de la orientación del contenido.

El marco teórico principal es la teoría de campos de Bourdieu, por su pertinencia con respecto a la producción cultural (Williams \& Garnham, 2003) y la lógica que subyace a las prácticas de los agentes productores y reproductores de cultura. Asimismo, se utilizan elementos de la teoría de mediatización para un análisis 
crítico de la interrelación entre los medios y las prácticas de comunicación, por un lado, y el cambio institucional, cultural y social por otro (Hepp \& Krotz, 2009).

\section{El periodismo educativo como campo}

Trascendiendo la perspectiva medio-céntrica enfocada exclusivamente en el texto/contenido (Couldry, 2012), la teoría de campos de Bourdieu proporciona un marco útil para analizar prácticas y disposiciones de producción cultural, tomando como punto de partida una perspectiva relacional (Bourdieu, 2003). Esto permite un balance entre agencia y estructura (Williams \& Garnham, 2003) y respalda un enfoque para problematizar las relaciones y equilibrar las perspectivas, alejándose de las opiniones dicotómicas que enfrenta la agencia humana con estructuras sociales (Hilgers \& Mangez, 2015).

Este paradigma relacional, que explora las prácticas, percepciones e inclinaciones de los individuos, se construye alrededor del concepto de campo (Bourdieu, 1995). Las entidades sociales se estructuran mediante una serie de campos jerárquicamente organizados, cada uno definido como un espacio estructurado, con sus propias leyes de funcionamiento y sus propias relaciones de fuerza. La teoría de campos permite analizar los mundos sociales específicos en los que se produce y reproduce conocimiento, prestando especial atención a las instituciones, relaciones y recursos materiales y simbólicos que los rodean (Benson \& Neveu, 2005). También rechaza un enfoque excesivamente culturalista, que retrata los sistemas simbólicos como ajenos a la estructura social (Benson \& Neveu, 2005).

Maares y Hanusch (2020) realizaron una revisión sistemática de 249 artículos que usaron la teoría de campos para investigar diferentes aspectos del periodismo. Notan su empleo para analizar la estratificación del campo periodístico, procesos de diferenciación, rutinas periodísticas, cambios normativos, estructuras sociales y relaciones de poder asociadas con el mundo periodístico, entre otros. Concluyen que, si bien aplicar la teoría de campos al análisis de la esfera periodística es promisorio, debe ser refinada, pues en el uso y apropiación de algunos conceptos de Bourdieu se cae en ambigüedades e imprecisiones.

\section{Teoría de campos y producción cultural}

Según Bourdieu (1995), cada campo es relativamente autónomo, pero estructuralmente homólogo con los demás. La autonomía depende de la medida en que el campo esté determinado por su propia lógica interna, o si es más susceptible a las influencias externas. Cuanto más autónomo sea el campo, más intenso será su poder de refracción y su capacidad para soportar arremetidas desde otros campos, o desde el campo general de poder (Bourdieu, 2000). Por el contrario, su heteronomía se manifiesta esencialmente cuando los eventos y campos adyacentes, especialmente económicos y políticos, se expresan e influyen directamente en él. 
Para Bourdieu, las clases hegemónicas buscan mantener su posición sobre el modo legítimo de producción cultural (Bourdieu, 1995), utilizando sus reservas de capital simbólico. Por su parte, el habitus orienta las acciones e inclinaciones de los individuos en el campo, proporcionando un sentido práctico de lo que es adecuado o no en un contexto específico (Bourdieu, 1977). El habitus se considera un tipo de constructivismo dialéctico, en el sentido de que toda práctica es producto de disposiciones previas, pero construidas en tensión permanente. La agencia humana en este paradigma es multidimensional, compleja y transformadora por las fuerzas contradictorias que componen los campos (Alonso, 2002). Los agentes generan estrategias según las fuerzas desplegadas en el campo (Bourdieu, 1990). El habitus se caracteriza porque sus disposiciones, generativas y transponibles, pueden influir en campos distintos de los adquiridos originalmente (Thompson, 2003).

Una de las fuerzas desplegadas en el campo es el capital simbólico, es decir el grado de prestigio acumulado, celebridad, consagración u honor, y se basa en una dialéctica de conocimiento y reconocimiento (Johnson, 1995). Mientras la lógica del capital económico es la ganancia, la del capital simbólico es la distinción. Las posiciones en los campos se componen por la diferencia y la dominación según la transferencia de diferentes capitales (Bourdieu, 1995). Cuando se establece un campo se entrecruzan las dinámicas expuestas anteriormente y comienza a ser gobernado por reglas implícitas, explícitas y principios de acción (Bourdieu, 1998), con personas o colectivos ocupando posiciones y orientadas a defenderlas o mejorarlas (Benson, 2006).

Para Bourdieu (1998), el campo periodístico se inserta en el campo general del poder, que a su vez se encuentra dentro del campo de la producción cultural. En el eje cultural o periodístico, son valorados elementos como la autonomía, los principios de la industria y el reportaje investigativo, opuestos a factores del eje económico como circulación, audiencia, publicidad y ganancias (Bourdieu, 1998, 2005; Benson \& Nevau, 2005). Otras investigaciones analizan campos dentro del campo periodístico. Duval (2005) mapea el periodismo económico, English (2006) el deportivo. Marchetti (2005) se centra en las especializaciones periodísticas para construir una serie de variables que expliquen sus diferencias.

\section{Educación y periodismo}

En términos de cobertura periodista educacional, algunos autores ponen el énfasis en la interacción entre la política pública educativa y el poder de los medios, destacando los efectos del periodismo en el debate educativo. Según Rawolle (2010), en el campo de la política educativa, la presencia de los medios es generalizada. Estas se diseñan e implementan acompañadas de comunicados de prensa y campañas 
publicitarias. Este autor utiliza la teoría de la mediatización para explicar la influencia de los medios en la política pública educativa, en instituciones o como prácticas, en otros campos de la estructura social (Livingstone \& Lunt, 2014). Los medios se vuelven cada vez más relevantes para construir la vida cotidiana, la sociedad y la cultura (Krotz, 2009).

Este trabajo se inserta también en la tradición de estudios de mediatización de la educación (Rawolle \& Lingard, 2010,2014) y Rawolle, 2005, 2010) ; que postula que hay vínculos específicos y verificables entre los procesos de globalización y la mediatización de la política pública educativa, denominados efectos de mediatización (Rawolle \& Lingard, 2014), relacionados con la promoción, distribución y adopción de conceptos de políticas. Así, la mediatización de la educación combina dos subprocesos: la configuración y el cambio de la política educativa para satisfacer las necesidades de las diferentes formas de periodismo, y la configuración y el cambio de la política educativa mediante el surgimiento de nuevas formas de tecnologías de la comunicación (Rawolle \& Lingard, 2014).

Para discutir la mediatización de la educación, Rawolle (2005) también emplea un enfoque Bourdeusiano. Argumenta que el cross-field effects son fenómenos y prácticas que no se pueden clasificar en un solo campo, sino que resultan de las interrelaciones entre campos. Concluye que entre la prensa y la política educativa existe una relación de impugnación, pero también simbiótica, con efectos estructurales y coyunturales entre ambos, lo que ha hecho, por ejemplo, que la política educativa deba someterse a ciertas lógicas y prácticas mediáticas.

De manera similar, Hjarvard (2017) señala que la investigación en mediatización debe considerar la interdependencia institucional entre medios y el campo a analizar. Así, no sólo existe la mediatización de la política, sino el proceso inverso de politización de los medios. Ejemplifica lo anterior usando además de la política el ámbito educativo, advirtiendo sobre una posible educationalization of media.

A nivel latinoamericano, Santa Cruz y Cabalín (2018) estudian la producción de noticias en Chile desde las opiniones de periodistas que cubren educación utilizando un marco Bourdesiano. Señalan que, aunque periodistas de educación reconocen que es un tema de alto interés para la élite, también tienden a minimizar o desconocer la naturaleza política de su trabajo, evitando cuestionar la influencia que podría tener su labor en la gobernanza educativa, y la legitimización de ciertas opiniones, narrativas y representaciones sociales. Es relevante señalar que se enfocan en periódicos, y que el campo del periodismo de educación es poco problematizado en sí mismo, visto como algo homogéneo, donde no se observan diferencias de opinión entre periodistas, luchas de poder, movilizaciones de capital, disputas por autonomía o tensiones con campos periodísticos cercanos. 
De la misma manera que el campo del periodismo está cruzado por las tensiones entre autonomía y usos de distintos capitales, el periodismo de educación, entendido como "aquella actividad periodística que contempla como su objetivo formal el contribuir a que la sociedad sepa y conozca objetivamente [...] ámbitos de la educación, enseñanza, formación, cultura reglada y no reglada, en todos los niveles académicos y etapas de la vida" (Crespo, 2005, p. 10) es una forma particular y especializada de construir noticias, con características, prácticas y fuentes propias distintas y distinguibles de otras formas periodísticas (García, 2018).

\section{METOdOLOGÍA}

Con el fin de caracterizar el campo del periodismo de educación desde la visión y prácticas de periodistas y editores, se utilizó un enfoque cualitativo interrelacionado e integrado (Flick, 2014). Se condujeron 24 entrevistas semiestructuradas en profundidad con profesionales de los medios, 19 periodistas y cinco editores/as, que cubren o que cubrieron educación en medios de comunicación en Chile. Estas se realizaronentre el 1 de marzo y el 15 de mayo de 2018.

Los participantes integran el campo del periodismo de educación de tres maneras:

- Cubren o cubrieron exclusivamente educación para el medio en que trabajan.

- Únicos encargados de reportear temas de educación, aunque en ocasiones otras temáticas.

- Editores/as exclusivos de la sección educación.

Se utilizó un muestreo intencional de informantes clave por bola de nieve, para tener acceso a detalles de otros casos ricos en información (Patton, 2002). Se identificaron 32 casos de periodistas y editores cubriendo educación en medios chilenos desde 2008 hasta mayo de 2018. De estos, 24 aceptaron ser entrevistados y trabajan o trabajaron en siete medios diferentes, con una o varias personas dedicadas a cubrir temas educativos. Para conocer qué medios tienen periodistas que cubran educación se realizaron las siguientes acciones:

- Buscar secciones denominadas "educación" o similares en sitios web de medios y periódicos en papel.

- Contacto con medios sin sección explícita y consulta por periodistas que cubrieran educación, sobre todo para radios.

- Pregunta a entrevistados por otros colegas y medios.

Se seleccionaron los siguientes medios: 
- Periódicos:

> El Mercurio (sección de educación).

> El Mercurio (sección política/nacional).

> La Tercera (sección de educación).

> La Tercera (sección política/nacional).

> Las Últimas Noticias (sección de educación).

La Tercera y El Mercurio tienen periodistas que cubren educación para la sección educación y para la sección política o nacional.

- Radios:

$>$ Cooperativa.

> Bío-Bío.

$>\mathrm{ADN}$.

- Sitios web de noticias:

> El Dínamo.

Se reconoce la dificultad de operacionalizar empíricamente los postulados de Bourdieu (Swartz, 1997), que el debate sobre qué constituye un campo está abierto (Friedland, 2009), y cómo emplear las herramientas conceptuales de la teoría de campos correctamente, así como sus límites (Stahl, 2016). Aun así, este estudio usa los conceptos de autonomía/heteronomía, habitus, capital simbólico y campo para generar subcategorías de operacionalización para construir las preguntas de las entrevistas (Seale, 2005). Algunas de estas subcategorías son: percepción del campo y de campos circundantes, experticia y especialización, luchas de poder, principios y rutinas profesionales, especificidades de lo educativo, selección de fuentes y temas, construcción de contenido, control, censura y autocensura, entre otros. Además, tomando en cuenta los estudios de mediatización, se indagó en efectos a través de los campos, influencias externas y lógicas mediáticas o institucionales. Así, por ejemplo, se preguntó por rutinas de trabajo, visión del trabajo propio, selección de temas y enfoques, relación con fuentes, trabajo con editores/as, percepción de campos cercanos, línea editorial y desafíos para cumplirla, entre otros.

Las entrevistas se analizaron con el enfoque temático crítico inductivo (Braun \& Clarke, 2006) buscando identificar, examinar yreportar patrones distinguibles dentro 
de los datos a partir de ideas recurrentes y unificadoras de las temáticas consultadas. La codificación se realizó utilizando categorías teóricas del marco conceptual.

\section{RESULTADOS}

\section{Orientación de contenido y dinámica del campo}

El campo, en general, tiene un nivel de autonomía variable. Para los entrevistados, "educación" es una fuente de noticias altamente predecible, pues durante el año hay hitos específicos regulares. Por ejemplo, el comienzo del año escolar, la comunicación de resultados de pruebas estandarizadas (SIMCE y PSU), el proceso de admisión a universidades, etc. Esto implica una rutina rígida con espacio limitado para la innovación o la iniciativa individual. Para muchos, esto implica que todos saben qué esperar, con las mismas contrapartes (universidades, Ministerio de Educación, etc.), y con enfoques similares. Estas noticias están dirigidas a una masa amplia e indeterminada y dependen en gran medida de factores externos. Por otro lado, se observó un alto grado de acuerdo entre los entrevistados sobre el hecho de que cuando las noticias de educación se cruzan con temas políticos hay una gama más amplia de opciones de cobertura. Por ejemplo, procesos de reforma educativa, cambios legislativos, opiniones del ministro/a de Educación, etc. Sin embargo, dada la naturaleza política del tema, dependen en gran medida del campo político y de la contingencia institucional.

Dados estos dos tipos de contenido, podría decirse que el campo debe posicionarse en el polo heterónomo. Sin embargo, esto puede ser problematizado, considerando otro tipo de contenido educativo mediático. Noticias sobre buenas prácticas en la enseñanza, programas de aprendizaje innovadores, o tecnología en las aulas son algunos ejemplos. Al no anclarse en la temporalidad educativa institucional y escapar a la discusión políticamente determinada, es un ejemplo de cierto grado de autonomía en el campo. Algunos periodistas expresaron que este tipo de noticias debería ser la esencia del periodismo educativo, lo que indicaría que su práctica es un tipo de capital simbólico. Quienes producen este tipo de contenido se sienten más cómodos con su propio trabajo y afirman estar haciendo lo que debería ser el periodismo de educación. Del mismo modo, quienes se centran en el contenido político, o contenido de hito educativo, expresaron que noticias sobre la escuela o el aula son ejemplos de periodismo educativo de calidad. Quienes lo hacen lo consideran especial, porque les da la oportunidad de estar en terreno, mostrar el mundo real de la educación e informar no solo sobre las políticas en educación, sino también su implementación por personas. Algunos(as) afirmaron que este tipo de periodismo requiere entender sobre pedagogía y aspectos técnico-educativos, lo que confiere un estatus especial a su práctica. 


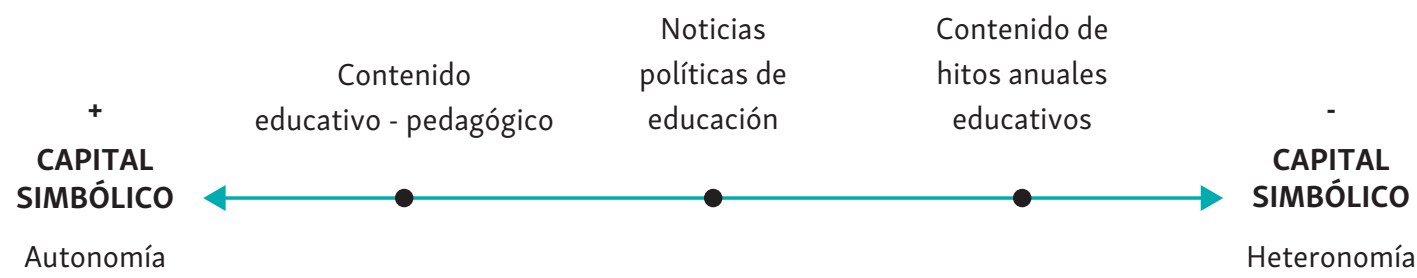

Figura 1. Tipos de contenido en periodismo educativo en el eje autonomía/heteronomía

Fuente: Elaboración propia.

Finalmente, para algunos de los entrevistados, los artículos sobre este tipo de temas no sólo consideran a un público amplio, sino a profesores, directores e investigadores. Aclaran que este tipo de noticias tiene efectos limitados en el público, en la imagen de los medios y entre los anunciantes. Se puede decir que, para este tipo de contenido, la lógica de nicho, típica de un campo autónomo, podría estar implícita

Por esta razón, es posible decir que el periodismo educativo tiene un nivel desigual de autonomía (figura 1). El contenido sobre noticias y reportajes pedagógicos, como en las noticias del aula y la escuela, tiene un mayor nivel de autonomía que el sobre política/política educativa o la agenda de hitos educativos. Los periodistas posicionados en el lado de la autonomía tienen más capital simbólico que sus colegas más cercanos del extremo de la heteronomía.

En términos de las menciones espontáneas a otros tipos de periodismo especializado, la más común fue el periodismo político, especialmente debido a una tensión detectada entre ese campo y el del periodismo de educación, que se abordará más adelante. Según los entrevistados, en términos de prácticas y comportamientos, ambos campos son muy similares en relación con las rutinas periodísticas, excepto en noticias sobre escuelas y aulas. Este tipo de contenido daría más libertad y autonomía al momento de reportear. Otros campos mencionados fueron el periodismo deportivo y el cultural. Afirman que las noticias deportivas tienen la mayor audiencia, alta presencia de vínculos comerciales y son las más atractivas para los anunciantes. Las secciones de cultura se mencionaron como un campo de nicho, especialmente cuando están relacionadas con las artes. Dada su especificidad y audiencia limitada, las asocian comúnmente con una audiencia de élite y pueden describirse como el campo con el más alto nivel de autonomía (figura 2). 


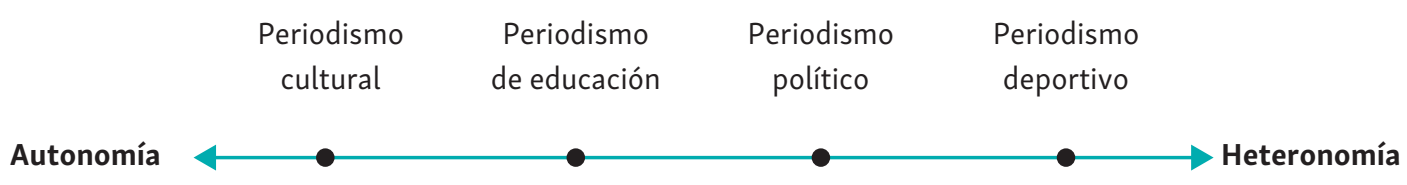

Figura 2. Otros campos del periodismo y su posición en el eje de autonomía/heteronomía

Fuente: Elaboración propia.

\section{Tipos de medios de comunicación y su posición en el campo}

Es relevante advertir que, tras el movimiento estudiantil de 2006, El Mercurio y La Tercera, dos de los periódicos más influyentes y de posición dominante en Chile (Navia \& Osorio, 2015; Santa Cruz, 2016), crearon una sección especial de educación con periodistas, según testimonios de entrevistados/as. Más tarde, otros medios incluyeron educación como una sección específica, con al menos un/a periodista especializado/a.

Significativamente, el análisis de las entrevistas reveló que en ocasiones el mismo medio puede clasificarse en diferentes lugares en el campo, según la forma en la cual se trata la información, lo que resulta en diversos grados de autonomía/ heteronomía. Lo anterior, considerando que en algunos medios el contenido sobre educación a veces se publica en la sección de educación y otras, en noticias generales o en la sección política.

Esto puede ejemplificarse en los periódicos El Mercurio y La Tercera. La sección de educación de El Mercurio se centra casi exclusivamente en artículos sobre experiencias en el aula, innovaciones en pedagogía, programas de aprendizaje y enseñanza y las consecuencias prácticas de implementar la reforma, entre otros. También publican entrevistas con académicos, expertos en educación, implementadores de programas y profesores. Noticias sobre políticas educativas, reforma educacional, etc., están cubiertas por periodistas que trabajan en la sección de política o nacional. En La Tercera, la sección de educación -desaparecida hace algunos años-, también cubría aspectos pedagógicos, pero tenía una mayor variedad de temas, otorgando asimismo espacio al ámbito político-institucional. Además, según testimonios de periodistas de este medio, las noticias relacionadas con aspectos políticos de la educación tuvieron más repercusión externa e impacto interno. Por ello podríamos situarlos más cerca de los polos de orientación política y heteronomía (figura 3). En consecuencia, las noticias generales sobre educación en ambos periódicos, publicadas por la sección política o nacional, se ubicarían en un cuadrante de orientación política y heteronomía. 
Con respecto a las estaciones de radio, se desprende del análisis que tanto en ADN y Radio Bío-Bío predomina un enfoque casi exclusivamente político. Los temas pedagógicos que surgen de las escuelas, aulas o universidades no son, en general, fuentes de noticias publicables. Los periodistas explican que esta ausencia es por las características de las noticias radiales, expresando que se alimentan de eventos actuales, basados en la inmediatez y dependientes de la temporalidad. Por ello, las noticias deben responder a lo que está ocurriendo y a temas sobre los cuales la gente está discutiendo. Por lo tanto, la cobertura se centra en cuestiones educativas imbricadas con la agenda política. Sin embargo, los entrevistados expresaron respeto por los periodistas de otros medios que publican artículos con orientación pedagógica. Afirman que esas noticias son valiosas y brindan información importante. Perciben que solo los periódicos tienen el tiempo y el espacio para lograr la profundidad y complejidad que requieren esos tipos de artículos. Esta característica de las radios es matizada en el caso de Radio Cooperativa. A pesar de la influencia de la agenda política de educación, su enfoque está levemente más inclinado hacia la orientación pedagógica. Periodistas de ese medio destacaron la producción de programas sobre temas educacionales/pedagógicos, especialmente sobre enseñanza, aprendizaje y buenas prácticas en entornos educativos. Es normal para ellos(as) ir a escuelas, hablar con maestros, estudiantes y padres para producir noticias. Es importante aclarar que este tipo de programación (Agenda Calidad, Buenas Escuelas, Elige Educar, entre otros) es financiada por ONGs y organismos gubernamentales, no necesariamente sujetos al financiamiento regular de avisadores.

La sección de educación del sitio web El Dínamo ofrece contenido que se inclina hacia el polo de orientación política. Sus informantes explicaron que sus contenidos, decisiones de selección y enfoque están más ligados a métricas web de visitas y permanencia. El contenido está definido por el contenido político, según sus testimonios, por rendir mejor dentro de la lógica digital y editorial, siendo ese el principal criterio de producción:

\section{"Cuando hay algo de política, nos aseguramos de que el tema pueda llegar a más gente. Generalmente diferencias políticas en educación tienen mejor llegada que cosas muy abstractas." (Periodista, sección educación).}

Finalmente, la cobertura periodística en educación del periódico Las Últimas Noticias se basa en artículos sobre educación terciaria y empleabilidad. Por ejemplo, retribución por estudios de pregrado o posgrado, clasificaciones sobre el costo de los programas de educación superior, carreras con mayor tasa de ocupación, etc. Según sus periodistas, brindan información relevante sobre su futuro a las personas que buscan oportunidades de educación. 


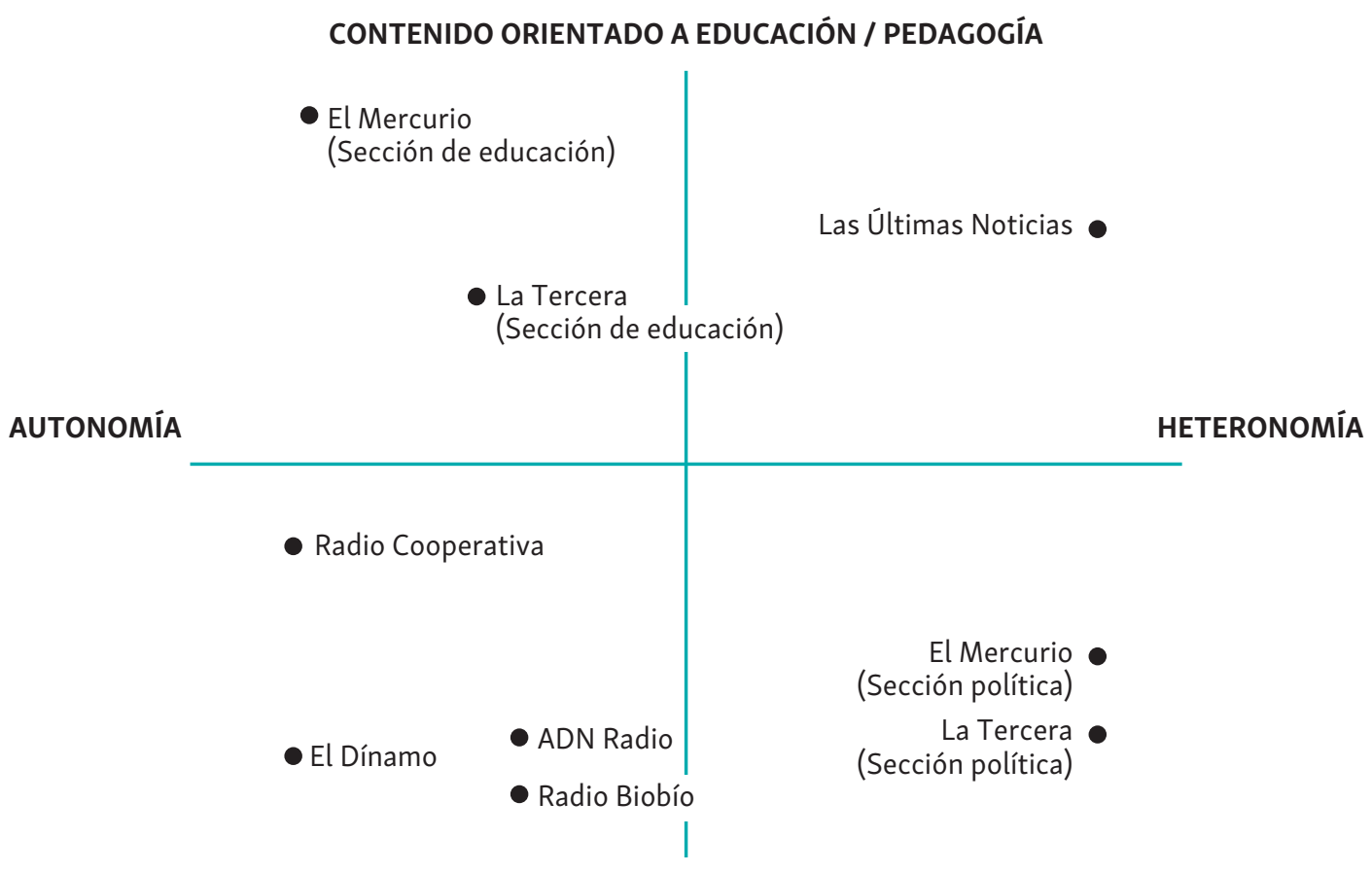

\section{CONTENIDO ORIENTADO A EDUCACIÓN POLÍTICA}

Figura 3. Posiciones en el campo según el tipo de contenido y nivel de autonomía Fuente: Elaboración propia.

También se desprende que este tipo de contenido es importante para proporcionar al mercado certezas sobre desarrollo profesional y empleabilidad. Es un contenido con un fuerte incentivo económico y sus fuentes de información (universidades e institutos técnicos) también son una parte importante de los ingresos del periódico a través de la publicidad. Por lo anterior, está situado en el eje de heteronomía. No es que en Las Últimas Noticias no se construyan noticias sobre el aspecto político o legislativo en educación, sino que no son producidas por periodistas exclusivamente dedicados a educación.

En síntesis, se puede decir que los periódicos pueden ubicarse en el eje de la heteronomía, aunque su sección de educación está mucho más cerca del polo de autonomía. Las radios están ubicadas en el eje de autonomía, pero solo en el segmento de orientación política (figura 3).

\section{Tensiones entre periodismo político y periodismo educativo}

Como se afirmó, una parte importante de las noticias sobre educación proviene de la agenda política. Todos los medios de los que formaron parte los participantes producen noticias sobre los aspectos políticos de la esfera educativa. En casi todos los casos de periodistas que cubren educación desde el polo de orientación política, se mencionó la tensión y el conflicto no resuelto con los periodistas que cubren política. 
En un primer nivel, el problema es sobre quién debería ser responsable de reportear historias donde no se distingue claramente el lado educativo y el político. En la mayoría de los casos no hay un criterio claro y definido para asignar temas y la decisión se toma caso a caso, lo que crea disputas y tensiones entre ambas partes. En otro nivel, el problema está relacionado con el tratamiento de la información cuando periodistas de política cubren estos temas. Para los periodistas de educación, sus colegas no están completamente preparados para comprender y comunicar adecuadamente los problemas educativos. Opinan que a menudo sólo informan sobre la superficie del problema, de manera superflua y descontextualizada.

"Los periodistas de política en general saben muy poco de la escuela, sobre todo los que están incorporados en crónica." (Periodista, sección educación).

“Era complicado separar temas. Yo veía temas de coyuntura y la sección de educación hacia análisis a largo plazo, algo que no fuera tanto del día. Pero no siempre resultaba la coordinación entre periodistas o entre editores. En las peticiones de temas nos cruzábamos." (Periodista, sección política).

Además, periodistas de las secciones educativas reclaman un exceso de atención en controversias y disputas políticas, sin considerar las consecuencias para el ámbito educativo. En este conflicto, demostrar conocimiento de los aspectos educativos es una forma de capital simbólico que se puede utilizar para adquirir estatus y ganar la asignación de historias y enfoques.

En algunos testimonios se aprecia que los periodistas de educación perciben un cierto desdén y un trato condescendiente por parte de los colegas de la sección política. Afirmaron que, a pesar de su experiencia y conocimiento sobre asuntos educativos, los periodistas de secciones políticas disfrutan de un mejor estatus y siempre están más cerca del poder en la organización, al ser más cercanos a quienes toman decisiones y tendrían mayor influencia y rango de acción:

"Política siempre fue el centro del diario, nosotros estábamos lejos. Éramos
considerados como sección de poca importancia y liviana, como cultura o
deporte. No senos tomaba mucho en cuenta." (Periodista, sección educación).

Según esas percepciones, el campo del periodismo político estaría mucho más cerca del campo general del poder que el campo del periodismo educativo.

\section{El campo y los lineamientos editoriales}

Según investigaciones anteriores (Cabalín, 2014b; Cabalín, 2015; Cabalín \& Antezana, 2016), los principales periódicos chilenos buscan reflejar las creencias político-económicas de sus propietarios mediante editoriales, artículos y portadas. Para explorar las características del campo y sus dinámicas internas es también 
esencial analizar cómo los periodistas abordan las posturas editoriales y entender cómo moldean sus prácticas y visiones.

Los periodistas de periódicos declararon que conocen, entienden y, en general, tratan de aplicar la línea editorial, incluso cuando en algunos casos no la compartan totalmente. En el caso de La Tercera, tanto en la sección política como en la educativa, los periodistas declararon que la posición política que el periódico quiere difundir es muy clara. Sin embargo, la manera de expresarla es sutil, no siempre directa y en ocasiones negociable, dependiendo del capital simbólico de cada periodista. Indicaron que la experiencia, habilidad negociadora o cuotas de poder en la organización, pueden ser también formas de apelar o contrarrestar decisiones editoriales que ellos creen equivocadas. Para los entrevistados de ese periódico, uno de los principios más mencionados por mandos superiores -editores de sección, generales o directores- es no olvidar que su audiencia objetivo es una determinada élite:

"Sabíamos que nuestro público casi siempre era una élite... Y el principal lineamiento era defender la libertad. En educación es la libertad de elegir los colegios, de conocer los resultados de sus hijos o de los colegios, o la libertad para saber resultados de la prueba de selección universitaria. Y en educación pública era dejar en claro que no todo lo público es solamente del Estado. También eso implicaba nombrar siempre a los privados. Si hablamos de educación superior tratar siempre de mencionar también universidades privadas e igual con los colegios." (Periodista, sección política).

Los periodistas de la sección educativa también mencionaron ese tipo de lineamientos, lo que fue complementado con los lineamientos editoriales según los gobiernos de turno:

"Si la derecha estaba en el gobierno teníamos que ser muy cuidadosos siempre y más cautos en todo lo que investigábamos y publicábamos. En cambio, con Bachelet (gobierno de centroizquierda), teníamos mucha más libertad para buscar problemas en sus políticas." (Periodista, sección educación).

En la sección política, afirman que la línea editorial de El Mercurio promueve ideas, espacio y voz sobre la importancia de la libertad de enseñanza y el papel relevante del emprendimiento privado en educación. Como ya se mencionó, la sección de educación casi no hace referencia a cuestiones políticas; por lo tanto, la línea editorial sería menos influyente en esta sección. Según informantes de esa sección, esta responde a un cierto sentido de misión, para contribuir a la educación chilena y cumplir con su responsabilidad social corporativa. Afirmaron que la línea editorial del diario, muy clara y conocida, es a veces imperceptible en la sección de 
educación. La omisión de una discusión política de temas educativos también puede considerarse una forma de reproducir la línea editorial de los medios, evitando debates que pueden conducir a una transformación del campo y, tal como plantea Cabalín (2014b), entender la educación como algo meramente técnico y no político también sería un discurso ideológico.

En el periódico Las Últimas Noticias, la línea editorial también excluye, según los entrevistados, los temas políticos de la sección de educación. La cobertura proporciona principalmente información sobre cuestiones de empleabilidad y nuevas oportunidades del tercer sector. Sin embargo, los testimonios indican que esta exclusión es reforzada permanentemente por los editores y directores, especialmente cuando se trata de información sobre las políticas educativas de un gobierno de izquierda:

"Nos dijeron que no publiquemos nada sobre gratuidad (política pública en educación superior). Tuvimos que bajar el tono y tratar de no promocionarlo. Por ejemplo, no podía poner la cifra de estudiantes que tienen educación gratuita. Nos dijeron que no era noticia, que deberíamos buscar otro enfoque. No se suponía que debíamos enfatizar que era bueno o que ayudaba a la gente." (Periodista, sección educación).

En radios, la situación es diferente y la producción de noticias presenta mayores grados de independencia. Todos los entrevistados expresaron que, incluso cuando los periodistas conocen la posición y las inclinaciones del propietario, estas no forman parte de la línea editorial. Según los participantes de radio Cooperativa, el único principio rector es la obligación de mostrar siempre ambos lados de la historia, ya sea en la voz de fuentes o como parte del relato.

Entrevistados de Radio Bío-Bío afirman que la única condición para informar es ser lo más transparentes y objetivos posibles, y que en general la radio no proporciona pautas o parámetros establecidos con respecto a cómo tratar los temas. Agregan que la línea editorial se expresa exclusivamente en artículos de opinión y comentarios editoriales, no así en el relato o encuadre noticioso.

En el caso de Radio ADN, los entrevistados expresaron que la línea editorial de la radio no ejerce ningún tipo de presión sobre su trabajo, pero la visión del rol y trabajo que ellos tienen como periodistas, está cargada de un discurso altamente político. De acuerdo con sus testimonios, es un deber denunciar las injusticias, desigualdades y anomalías del sistema educativo chileno, especialmente en relación con el creciente proceso de privatización y mercantilización. De acuerdo a sus opiniones, editores y directores nunca han tratado de disuadirlos de ese enfoque. 
Con respecto al sitio web de noticias El Dínamo, los entrevistados expresaron que la línea editorial del medio promueve publicar contenido atractivo e interesante, que, en el caso de cuestiones educativas, se traduce en artículos que representen controversias y conflictos ideológicos entre actores educativos, especialmente políticos. En este caso, afirman, el periodista cubre la disputa, tratando de mostrar imparcialidad y neutralidad, a menos que sea un asunto en el que una posición es tan obviamente impopular con la mayoría de la audiencia que una inclinación sutil ayudará a aumentar las visitas.

\begin{abstract}
“Tratamos de mostrar las diferencias políticas que hay en educación, pero igual tratando de ser constructivos. Nos interesa que se vean las diferentes posturas y que la educación no da lo mismo, pero obviamente tratamos de ser objetivos y serios. Claro, a menos que sea algo muy loco, en que obviamente hay que tomar partido para llegar mejor a la audiencia." (Periodista, sección educación).
\end{abstract}

\title{
Lógica de medios y mediatización
}

Respecto de la selección de temas, las diferencias, nuevamente, se basan en el tipo de medios y su orientación. Así, por ejemplo, los periodistas de radio seleccionan los temas utilizando un método personal, casi siempre de acuerdo con sus propios criterios. En el caso de la sección política en los periódicos, también. Sin embargo, los entrevistados señalan que es común que el editor u otras autoridades superiores propongan temas.

En cuanto al tratamiento de la información, la mayoría de los periodistas de las secciones de educación se quejaron del contenido educacional de otros medios, especialmente de los que no tienen secciones de educación ni periodistas especializados. Estiman que, en general, la cobertura carece de profundidad y de un enfoque integral y amplio. Sin embargo, es interesante notar que algunas de las críticas son autoinfligidas. Alrededor de 10 periodistas, sobre todo de medios escritos, mencionaron que cuando publicaron información cuantitativa, expresada en clasificaciones y rankings de desempeño, no contribuyeron a la discusión educativa en Chile. Para ellos, especialmente para los periodistas de la sección de educación de La Tercera, una práctica común, e incluso un objetivo, era tratar de producir artículos basados en la interpretación de datos empíricos, especialmente sobre indicadores de aprendizaje y tipos de sostenedores. Por lo tanto, reportajes sobre cómo el sistema privado obtenía mejores resultados de aprendizajes, artículos sobre la clasificación de escuelas, o notas sobre los mejores colegios de Chile se hicieron comunes y tuvieron gran impacto entre el público. Después de unos años, las voces de la academia y el sector público (Equipo de Tarea Comisión SIMCE, 2014) comenzaron a criticar este tipo de cobertura, por caer en descontextualizaciones, simplificar problemas complejos, ser metodológicamente imprecisa al comparar y 
especialmente por su peligro en la estigmatización de ciertos tipos de escuela. Este reproche es compartido por la mayoría de los periodistas y editores de periódicos, y la mayoría recalca que intentan no reproducir ese tipo de contenido:

"En el momento en que sacamos los rankings fue una necesidad de que las instituciones rindieran cuenta y la gente supiera qué pasaba en el mercado. Suplir la falta de información. Con el tiempo me fui dando cuenta que el tema se usó tanto que al final los colegios terminaron haciendo sus cosas en virtud de cómo salían en los rankings. Fue peor. Le hicimos un flaco favor a los colegios, porque les importaban los rankings PSU y SIMCE. Fue contraproducente para que todos los alumnos salieran adelante y no solo algunos. Los medios ejercieron una influencia negativa en el sistema escolar. Porque, además, los rankings no presentan todas las variables y solo era rendimiento, pero hay otras que no se pueden medir y que son importantes" (periodista de La Tercera, sección educación).

Sin embargo, algunos expresaron que en ocasiones es necesario, ya sea porque los editores lo solicitan o porque es contenido comprobado con buena aceptación por la audiencia.

En este caso, podemos observar la materialización de un cross-field effect en el proceso de mediatización de la educación (Rawolle, 2005), o cómo las lógicas del campo periodístico afectan directamente al campo educativo. Inversamente, con el ejemplo de periodistas que cambian sus prácticas y visiones siguiendo recomendaciones de expertos en educación sobre el mal uso de rankings y mediciones, podríamos teorizar tal fenómeno, siguiendo a Hjarvard (2017), como un caso de educationalization of media, es decir cómo el campo de la educación también influye y afecta al campo de los medios y el periodismo.

\section{CONCLUSIONES}

La percepción de los editores y periodistas entrevistados muestra que el campo del periodismo de educación no es homogéneo. Existen diferencias en el tipo de noticias generadas por los periodistas, en el nivel de autonomía y heteronomía que experimentan y en cómo se relacionan con los lineamientos editoriales. Esas diferencias pueden explicarse por sus posiciones en el campo, niveles de capital simbólico, y el conjunto de disposiciones que movilizan su actuar según entienden la educación en el país (habitus). Los medios con foco en noticias sobre educación tienen niveles más altos de autonomía que los con énfasis político. En términos de campos de periodismo circundantes, para los entrevistados el periodismo educativo es más autónomo que el periodismo político o deportivo, pero menos que el periodismo cultural. Considerando el tipo de medios, la investigación muestra 
que los periódicos pueden posicionarse en el cuadrante de la heteronomía, mientras la radio tiene niveles más altos de autonomía.

Un resultado importante es la relación problemática entre los periodistas de educación (especialmente aquellos con enfoque en contenido político) y los periodistas políticos que cubren educación. Del análisis se desprende que los periodistas de educación tienen un cierto habitus construido en torno al discurso sobre la importancia de la educación para la sociedad y la práctica de un periodismo involucrado y responsable del desarrollo del país y sus familias. Esta narrativa la construyen tomando como base la experiencia y el conocimiento técnico necesarios para reportar problemas educativos.

Con respecto a cómo el trabajo del periodista de educación se ve afectado por los sesgos políticos del entorno mediático chileno, los testimonios muestran que los periódicos tienen una línea editorial clara. En muchas ocasiones está presente en forma de mandato para seleccionar y tratar el contenido, mientras que las radios tienen más libertad para no reproducir las opiniones de los propietarios.

Esta investigación muestra que las lógicas de producción de noticias son una fuente de conflicto y preocupación entre los periodistas de educación. Son conscientes de que la simplificación excesiva, usar estadísticas descontextualizadas y enfocarse en conflictos y luchas de poder pueden perjudicar el campo educativo y una mejor comprensión de la política pública educativa por la ciudadanía. También se detalla la existencia, desde la visión de periodistas y editores, de dinámicas tipo cross-field, en las que las lógicas mediáticas podrían afectan el campo educativo; también podría darse el fenómeno inverso, en el que elementos del discurso educativo afectan la práctica periodística (educalization of media).

Como trabajo exploratorio, este estudio es un primer esfuerzo por comprender mejor las dinámicas que ocurren al interior del campo del periodismo de educación y cómo sus propios actores lo entienden y construyen. Sin embargo, al ser la teoría de campos fundamentalmente relacional, la exploración de campos circundantes también es relevante. Así, un límite de esta exploración está dada por la falta de voces y visiones de otros campos relacionados. Por ejemplo otros periodismos especializados o incluso campos ligados a la educación, como instituciones gubernamentales, escuelas, universidades o diseñadores de políticas públicas. A su vez, el punto de vista de la recepción del contenido está ausente, sobre todo las posibles consecuencias de las interpretaciones de la audiencia y su retroalimentación para generar noticias sobre educación. Finalmente, existe una amplia tradición investigativa sobre identidades, roles y cultura periodística (Hanitzsch, 2017; Hanitzsch \& Vos, 2017; Mellado, 2019) que podría usarse en conjunto con la teoría de campos, la cual no formó parte de este estudio, pero que podría ser relevante para futuras investigaciones. 


\section{REFERENCIAS}

Alonso, L. E. (2002). Pierre Bourdieu" in memoriam"(1930-2002): Entre la Bourdieumanía y la reconstrucción de la sociología europea (Pierre Bourdieu "In Memoriam" (1930-2002): Between Bourdieumania and the reconstruction of European sociology). Reis, (97), 9-28. Retrieved from https://www.jstor.org/stable/40184363

Arredondo, C. P. (2016). La representación visual del movimiento estudiantil chileno en la prensa establecida y alternativa nacional: Un análisis multimodal (The visual representation of the Chilean students' movement in the national established and alternative press: A multimodal análisis). Revista Austral de Ciencias Sociales, (30), 5-26. https://doi.org/10.4206/rev.austral.cienc.soc.2016.n30-01

Bellei, C. \& Cabalín, C. (2013). Chilean student movements: Sustained struggle to transform a market-oriented educational system. Current Issues in Comparative Education, 15(2), 108-123. Retrieved from https://www.tc.columbia.edu/cice/pdf/28175_15_02_Bellei_Cabalin.pdf

Bellei, C., Cabalín, C., \& Orellana, V. (2014). The 2011 Chilean student movement against neoliberal educational policies. Studies in Higher Education, 39(3), 426- 440. https://doi.org/10.1080/03075079.2014.896179

Benson, R. (2006). News Media as a "Journalistic Field": What Bourdieu Adds to New Institutionalism, and Vice Versa. Political Communication, (23)2, 187-202. https://doi.org/10.1080/10584600600629802

Benson, R. \& Neveu, E. (2005). Bourdieu and the journalistic field. Polity.

Bourdieu, P. (1977). Outline of a Theory of Practice. Cambridge University Press.

Bourdieu, P. (1990). The logic of practice. Stanford University Press.

Bourdieu, P. (1995). The field of cultural production: Essays on art and literature.: Columbia University Press.

Bourdieu, P. (1998). On television. The New Press.

Bourdieu, P. (2000). Los usos sociales de la ciencia (The Social Uses of Science). Nueva visión.

Bourdieu, P. (2003). Language and symbolic power, ed. by J. B. Thompson. Harvard University Press.

Braun, V. \& Clarke, V. (2006). Using thematic analysis. Qualitative Research in Psychology, 3(2), 77-101. https://doi.org/10.1191/1478088706qp063oa

Cabalín, C. (2013). Framing y políticas educacionales: Los medios como actores políticos en educación (Framing Educational Policies: The media as political actors in education). Estudios sobreel Mensaje Periodístico, 19(2), 635-647.https://doi.org/10.5209/rev_ESMP.2013.v19.n2.43463

Cabalín, C. (2014a). Estudiantes conectados y movilizados: El uso de Facebook en las protestas estudiantiles en Chile (Online and mobilized students: The use of Facebook in the Chilean student protests). Comunicar, (43), 25-33.https://doi.org/10.3916/C43-2014-02

Cabalín, C. (2014b). The conservative response to the 2011 Chilean student movement: Neoliberal education and media. Discourse: Studies in the Cultural Politics of Education, 35(4), 485-498.https://doi.org/10.1080/01596306.2013.871233 
Cabalín, C. (2015). The mediatization of educational policies in Chile: The role of the media in a neoliberal education field. In Z. C. Wubbena, D. R. Ford, \& B. Porfilio (Eds.), News media and the neoliberal privatization of education (pp.9-22). Charlotte, NC: Information Age Publishing.

Cabalín, C. \& Antezana, L. (2016). La educación en portada: la visualización de la política educacional en la prensa (Education cover page: The visualization of educational policy in the press). Cuadernos.Info, (39), 195-207.https://doi.org/10.7764/cdi.39.914

Cabalín, C., Montero, L., \& Cárdenas, C. (2019). Discursos mediáticos sobre la educación: el caso de las pruebas estandarizadas en Chile (Media discourses about education: the case of standardized tests in Chile). Cuadernos.Info, (44), 135-154.https://doi.org/10.7764/cdi.44.1429

Couldry, N. (2012). Media, society, world: Social theory and digital media practice. Polity.

Crespo, D. (2005). Periodismo educativo (Educational journalism). Síntesis.

Duval, J. (2005). Economic journalism in France. In R. Benson \& E. Neveu (Eds.), Bourdieu and the journalistic field (pp. 135-155). Cambridge, England.

English, P. (2016). Mapping the sports journalism field: Bourdieu and broadsheet newsrooms. Journalism, 17(8), 1001-1017https://doi.org/10.1177/1464884915576728

Equipo de Tarea Comisión SIMCE. (2014). Hacia un sistema completo y equilibrado de evaluación de los aprendizajes en Chile (Towards a complete and balanced system for evaluating learning in Chile). Retrieved fromhttps://www.mineduc.cl/wp-content/uploads/ sites/19/2015/11/Informe-Equipo-de-Tarea-Revisi\%C3\%B3n-Simce.pdf

Figueroa, F. (2013). Llegamos para quedarnos. Crónicas de la revuelta estudiantil (We are here to stay. Chronicles of the student revolt). LOM Ediciones.

Flick, U. (2014). The SAGE Handbook of Qualitative Data Analysis. SAGE.

Friedland, R. (2009). The Endless Fields of Pierre Bourdieu. Organization, 16(6), 887-917. https://doi.org/10.1177/1350508409341115

García, H. (2018). El periodista educativo y sus circunstancias: lo que el lector no ve (The Education Journalist and His Circunstances: What the Reader Can't See). Revista de Sociología de la Educación-RASE, 11(3), 393-404. https://doi.org/10.7203/RASE.11.3.13031

García Canclini, N. (1984). Gramsci con Bourdieu. Hegemonía, consumo y nuevas formas de organización popular (Gramsci with Bourdieu. Hegemony, consumption, and new forms of popular organization). Nueva Sociedad, (71), 69-78. Retrieved from https://static.nuso. org/media/articles/downloads/1156_1.pdf

Hanitzsch, T. (2017). Professional Identity and Roles of Journalists. Oxford Research Encyclopedia of Communication. Oxford University Press.https://doi.org/10.1093/acrefore/9780190228613.013.95

Hanitzsch, T. \& Vos, T. P. (2017). Journalistic Roles and the Struggle Over Institutional Identity: The Discursive Constitution of Journalism: Journalistic Roles and Institutional Identity. Communication Theory, 27(2), 115-135. https://doi.org/10.1111/comt.12112

Hepp, A. \& Krotz, F. (Eds.). (2014). Mediatized worlds: Culture and society in a media age. Springer.

Hilgers, M. \& Mangez, E. (2015). Bourdieu's theory of social fields: Concepts and applications. Routledge. 
Hjarvard, S. (2017). Mediatization. In C. Hoffner and L. Zoonen (Eds.), The International Encyclopedia of Media Effects. Wiley https://doi.org/10.1002/9781118783764.wbieme0107

Jiménez, C. \& Muñoz, J. (2008). Estructura de los medios de comunicación en Chile (Media structure in Chile). Razón y Palabra, (60).

Johnson, R. (1995). Editor's Introduction: Pierre Bourdieu on Art, Literature and Culture. In P. Bourdieu, Essays on art and literature, ed. by R. Johnson (pp. 1-25). Columbia University Press.

Krotz, F. (2009). Mediatization: A concept with which to grasp media and societal change. In K. Lundby (Ed.), Mediatization: Concepts, Changes, Consequences (pp. 21-40). Peter Lang.

Livingstone, S. \& Lunt, P. (2014). Mediatization: an emerging paradigm for media and communication research? In K. Lundby (Ed.), Mediatization of Communication (pp. 703-724). De Gruyter. https://doi.org/10.1515/9783110272215.703

Maares, P. \& Hanusch, F. (2020). Interpretations of the journalistic field: A systematic analysis of how journalism scholarship appropriates Bourdieusian thought. Journalism, 1-19. https://doi.org/10.1177/1464884920959552

Marchetti, D. (2005). Subfields of specialized journalism. In R. Benson \& E. Neveu (Eds), Bourdieu and the journalistic field (pp.64-84). Polity Press.

Mellado, C. (2019). Journalists' Professional Roles and Role Performance. Oxford Research Encyclopedia of Communication. Oxford University Press.

https://doi.org/10.1093/acrefore/9780190228613.013.832

Navia, P. \& Osorio, R. (2015). El Mercurio Lies, and La Tercera Lies More. Political Bias in Newspaper Headlines in Chile, 1994-2010. Bull Lat Am Res, 34(4), 467-485. https://doi.org/10.1111/blar.12364

Patton, M. Q. (2002). Qualitative Research and Evaluation Methods (3rd ed.). Sage.

Rawolle, S. (2005). Cross-field effects and temporary social fields: A case study of the mediatization of recent Australian knowledge economy policies. Journal of Education Policy, 20(6), 705-724. https://doi.org/10.1080/02680930500238622

Rawolle, S. (2010). Understanding the Mediatisation of Educational Policy as Practice. Critical Studies in Education, 51(1), 21-39. https://doi.org/10.1080/17508480903450208

Rawolle, S. \& Lingard, B. (2010). The mediatization of the knowledge based economy: An Australian field based account. Communications, 35(3), 269-286. https://doi.org/10.1515/comm.2010.015

Rawolle, S. \& Lingard, B. (2014). Mediatization and education: a sociological account. In K. Lund (Ed.), Mediatization of Communication (pp. 595-616). De Gruyter. https://doi.org/10.1515/9783110272215.595

Santa Cruz, E. (2016). Mediatización de las políticas educativas en Chile: El discurso de los diarios La Tercera y El Mercurio sobre la Ley General de Educación (2006-2009) (Mediatization of educational policies in Chile: The discourse of the newspapers La Tercera and El Mercurio on the General Education Law (2006-2009)). Doctoral dissertation, Universidad de Granada. Retrieved from http://hera.ugr.es/tesisugr/26082603.pdf 
Santa Cruz, E. \& Cabalín, C. (2019). Making Education News in Chile: Understanding the Role of Mediatization in Education Governance through a Bourdieuian Framework. In A. Wilkins \& A. Olmedo (Eds.), Education Governance and Social Theory: Interdisciplinary Approaches to Research (pp. 159-174). Bloomsbury Academic. https://doi.org/10.5040/9781350040090.ch-008

Saavedra Utman, J. (2019). The media commons and social movements: Grassroots mediations against neoliberal politics. Routledge.

Seale, C. (2005). Researching society and culture (2nd ed.). SAGE.

Stahl, G. (2016). Doing Bourdieu justice: thinking with and beyond Bourdieu. British Journal of Sociology of Education, 37(7), 1091-1103.https://doi.org/10.1080/01425692.2016.1216819

Sunkel, G. \& Geoffroy, E. (2001). Concentración económica de los medios de comunicación (Economic concentration of the media). Lom Ediciones.

Swartz, D. (1997). Culture and Power: The Sociology of Pierre Bourdieu. University of Chicago Press. https://doi.org/10.7208/chicago/9780226161655.001.0001

Thompson, J. B. (2003). Editor's Introduction. In P. Bourdieu, Language and symbolic power(pp1-31).r, ed. by J. B. Thompson.: Harvard University Press.

Williams, R. \& Garnham, N. (2003). Pierre Bourdieu and the sociology of culture: An introduction. In Design and Aesthetics (pp.59-72). Routledge.

\section{SOBRE EL AUTOR}

SEBASTIÁN ANSALDO, Periodista y magíster en Comunicación de la Universidad Diego Portales (Chile) y MPhil en Sociología de la Universidad de Cambridge (Reino Unido). Candidato a doctor en Educación por la Universidad de Cambridge. Interesado en las intersecciones e interacciones multidisciplinarias entre medios/comunicación y el campo educativo. Miembro del clúster de investigación Knowledge, Power and Politics de la Universidad de Cambridge y del Cambridge Latin American Research in Education Collective (CLAREC).

(iD) https://orcid.org/0000-0001-6483-5478 